\section{$\underset{\substack{\text { hommes } \\ \text { \& migrations }}}{ }$}

\section{Hommes \& migrations}

Revue française de référence sur les dynamiques

migratoires

$1315 \mid 2016$

Ondes de choc

\title{
Le (mauvais) esprit des lois
}

\section{Mustapha Harzoune}

\section{CpenEdition \\ Journals}

\section{Édition électronique}

URL : http://journals.openedition.org/hommesmigrations/3739

DOI : 10.4000/hommesmigrations.3739

ISSN : 2262-3353

\section{Éditeur}

Musée national de l'histoire de l'immigration

\section{Édition imprimée}

Date de publication : 1 juillet 2016

Pagination : 130-133

ISBN : 978-2-919040-36-0

ISSN : $1142-852 X$

\section{Référence électronique}

Mustapha Harzoune, «Le (mauvais) esprit des lois », Hommes \& migrations [En ligne], 1315 | 2016, mis en ligne le 02 janvier 2017, consulté le 15 septembre 2020. URL : http://journals.openedition.org/ hommesmigrations/3739 


\section{KIOSQUE}

\section{LE (MAUVAIS) ESPRIT DES LOIS}

Par MUSTAPHA HARZOUNE

e quinquennat qui se termine aura au moins _eu un mérite : éviter de faire de l'immigration un repoussoir électoral. Depuis 2012, la majorité parlementaire et l'exécutif ont épargné à leurs concitoyens les annuelles danses de Saint-Guy des politiques du chiffre, le carnaval de l'identité nationale, la danse du ventre des fiers-à-bras, postures et coups de menton télégéniques ad hoc, le tout contribuant à faire de la peur et de la suspicion les deux mamelles d'une politique à la vue basse et le plus sûr ferment des divisions et peut-être des déchirements du corps social. Pour autant, comme il convient de ne pas heurter une opinion présumée revêche, la majorité a adopté un profil bas, s'abstenant de toute politique nouvelle et innovante. Elle a assuré le service minimum en restant dans les clous d'une politique qui, bon an mal an, est la même depuis plus de quarante ans et qui pourrait se résumer en une volonté affichée, revendiquée, de contrôle de l'immigration. Droite dure ou gauche molle, cette politique, qui est une illusion sur le plan statistique, débouche sur des pratiques aux conséquences dangereuses comme vient de le rappeler le Défenseur des droits dans un rapport sur les droits des étrangers en France publié le 9 mai : "Les différentes lois relatives aux conditions d'entrée et de séjour en France des étrangers ayant succédé à l'ordonnance du 2 novembre 1945 ont, à de rares exceptions près, conduit à une régression de la situation des étrangers (...). Ce faisant, elles ont renforcé la banalisation, dans les esprits et dans le droit, du traitement différencié des individus à raison de leur nationalité. " Rappel d'une règle martiale par Jacques Toubon en charge depuis le
17 juillet 2014 de l'institution : l'important n'est pas la taille des biscoteaux, mais la qualité de l'ancrage, autrement dit la solidité des positions : entendre le respect des valeurs qui fondent le commun républicain.

Oui, depuis bientôt cing ans, la figure de l'immigré ne fut pas - pas du fait des responsables politiques en tout cas - pétrifiée sous le halo des projecteurs médiatico-politiques. Pour autant, il en est en Europe qui n'ont pas hésité à aller plus loin, à braver électeurs, opinions et consciences. Angela Merkel s'est engagée sur une politique autrement courageuse et le pape (et oui !) rappelle qu'un chat est un chat. En France, la gauche a du mal à retrouver ses petits... Si la fièvre semble être retombée, la guérison n'est pas à l'ordre du jour tant l'ignorance, la peur et la suspicion continuent de contaminer les débats (et l'année électorale qui pointe n'annonce rien qui vaille). Pire, les atteintes au droit persistent et avec elles la santé démocratique du pays chancelle. En effet, pour le Défenseur des droits, "le respect des droits des étrangers est un marqueur essentiel du degré de protection et d'effectivité des droits et libertés dans un pays ". Dans ce rapport sur le droit des étrangers, il relève "l'ensemble des obstacles qui se dressent à l'accès des étrangers aux droits fondamentaux en France et mesurer l'écart entre les droits proclamés et les droits effectivement exercés". Or ces obstacles ne relèvent pas de seules pratiques dépourvues de base légale. "C'est dans la règle de droit elle-même qu'une tension forte existe entre la proclamation et la réalisation d'un "principe universaliste d'égalité", qui conduit à supprimer les différences de traitements 
illégitimes, et d'un "principe réaliste de souveraineté étatique" qui conduit à créer et développer des régimes juridiques et un accès aux droits différents fondé sur la nationalité. "

Déjà, dans Le Monde du 4 février 2016, dans un contexte de lutte contre le terrorisme, Jacques Toubon s'inquiétait que l'" on entre dans l'ère des suspects". "Il est de la responsabilité du Défenseur des droits, et je l'ai dit dès le lendemain des attentats de janvier 2015, de rappeler le droit et de rappeler aux libertés dans le souci de maintenir la cohésion sociale et l'équilibre entre sécurité et liberté. L'union dans la peur et l'objectif de sécurité, c'est du court terme. La cohésion du pays est un enjeu de long terme. Cette parole doit être portée quels que soient les sondages et les majorités parlementaires. II ne faut pas baisser la garde face au terrorisme, mais c'est du maintien des exigences de notre démocratie dont je parle, pas d'une arme de guerre prête à tirer. "

Quant à l'écart entre la proclamation des droits dont bénéficient les étrangers, migrants ou non, et leur mise en œuvre, il déclarait : "Notre mission cardinale est d'éviter le "à quoi bon" de personnes qui se sentiraient abandonnées par la communauté nationale. Soit parce qu'elles ignorent leurs droits, soient parce qu'elles ne savent pas à qui s'adresser. L'accès au droit de tous et la capacité des différents services publics à leur apporter une réponse sont un élément de cohésion nationale. Les 400 délégués du Défenseur des droits sur le terrain offrent une grande proximité pour s'attaquer à ce phénomène de non-recours alors que les dénis de droits, face à l'administration, dans les situations de discrimination, mettent en cause l'égalité, qui est le principe de la République et le combat du Défenseur des droits. »

\section{L'égalité sous conditions}

En répertoriant " ces dénis de droits ", le rapport plonge dans le "brut » et parfois le brutal du quotidien de l'étranger. Ce faisant, il met en garde contre un danger plus grand encore et qui menace l'ensemble de la société, celui de fragiliser les principes qui fondent son unité, à commencer par l'égalité. "Les étrangers bénéficient, en principe, d'une égalité de traitement avec les nationaux, la nationalité devenant un critère prohibé de discrimination. Pourtant, les objectifs de maitrise de l'immigration conduisent à ce que cette égalité soit mise à mal, tant par les pratiques ouvertement illégales que par des lois qui entravent l'accès aux droits fondamentaux des étrangers. "

C'est donc une longue liste des atteintes à ces droits fondamentaux qui fait le ciel noir de l'étranger, de fraîche date ou non, en matière d'entrée et de séjour : liberté d'aller et venir, de circulation sur le territoire, droit au mariage ou à l'ouverture d'un compte en banque, contrôles d'identité qui, dans une logique de contrôle migratoire, ciblent « les étrangers ou les individus présumés comme tels", discriminations, légitimées par le droit interne, quant à l'accès aux minima sociaux conditionné par une obligation de résidence fixée à l'égard des seuls étrangers. À cela s'ajoutent les "restrictions d'accès à l'emploi ", à la santé au logement et une mention particulière pour les droits spécifiques des mineurs ou ceux des demandeurs d'asile.

Exemples concrets. Le droit au respect de la vie privée et familiale se heurte aux refus de visas opposés sur le motif du « risque migratoire » à des parents de Français souhaitant rendre visite à leurs enfants et petits-enfants français. De même la délivrance des attestations d'accueil est subordonnée par " certaines mairies à des exigences non prévues par les textes ». Dans les préfectures, à l'indignité des conditions d'accueil s'ajoute l'atteinte " au droit des étrangers à voir examiner leur situation " par des délais de traitement excessifs, par d'importantes lacunes dans l'information délivrée aux usagers ou encore la non-délivrance des récépissés de demande de titre autorisant le demandeur, le cas échéant, à travailler.

Côté droits des mineurs, le Défenseur des droits dénonce les refus de scolariser des enfants dont 


\section{KIOSQUE}

les parents ne sont pas en séjour régulier ou sont sans domicile, alors que la scolarisation d'un enfant entre 6 ans et 16 ans est obligatoire en France. Idem à propos des mineurs qui sont de plus en plus nombreux à être placés en centre de rétention avec le reste de leur famille.

En matière d'accès aux soins, le rapport rappelle que le droit à la protection de la santé, impose aux autorités des obligations positives, or, en la matière, concernant les demandes d'AME (Aide médicale de l'État), il note des « contrôles longs et excessifs des conditions nécessaires d'ouverture des droits, ainsi que des demandes de documents injustifiées". Toujours selon ce rapport, "les obstacles à l'admission au séjour pour soins sont de plus en plus fréquents. Les éloignements des personnes porteuses du VIH vers le Nigeria ou le Surinam marquent une régression qui illustre l'inversion des priorités entre la "maîtrise" des flux migratoires et le respect des droits fondamentaux, dont le droit à la vie ". Rien de moins.

Les mesures d'éloignement sont aussi et parfois exécutées au mépris de certains droits fondamentaux, comme le respect de la vie privée et familiale, la prohibition des traitements inhumains ou dégradants, l'intérêt supérieur de l'enfant ou encore le droit des victimes à porter plainte - citant en l'occurrence l'interpellation et la reconduite à la frontière vers le Maroc "d'une jeune femme en situation irrégulière venue porter plainte pour violence ".

Le Défenseur des droits rappelle que le droit d'émigrer constitue un cadre juridique protecteur, notamment pour des personnes fuyant des persécutions. Or " la politique de gestion des frontières menée au niveau européen et national » compromet "l'effectivité » de ce droit. "Depuis plusieurs années, l'UE pratique une politique de "gestion concertée" des flux migratoires par la mise à contribution de pays tiers et l'externalisation des demandes de protection inter- nationale, comme avec la Turquie. La France tend à réduire les voies d'immigration légales, alors même que la situation en Syrie accroit la pression migratoire."

\section{La pente de la «préférence nationale »}

Pas étonnant dès lors que Jacques Toubon " dénonce un discours politique trop tourné vers la question "identitaire" " (site Senat 360 sur Public Sénat, le 9 mai) : "Cet agenda unique de l'identité fait qu'en réalité il n'y a plus aujourd'hui qu'une seule chose à faire: distinguer entre nationaux et étrangers, au mépris des principes. "Et de continuer: "Je rappelle que l'État est souverain et il peut faire des règles différentes quand il s'agit de l'entrée du séjour ou de l'éloignement des étrangers. Que ces lois soient trop restrictives aujourd'hui je le dénonce. Mais quand il s'agit des droits fondamentaux, des droits sociaux, des droits à la santé, des droits à l'éducation... Les droits sont universels et on ne peut pas les distinguer entre nationaux et étrangers. " Le Défenseur des droits ne manque pas de rappeler l'un des fondements du contrat républicain : l'égalité. Et c'est après avoir été saisi à de multiples reprises de cas de rupture d'égalité en défaveur des étrangers que Jacques Toubon a décidé d'initier ce rapport. "Mon but avec ce rapport n'est pas de dénoncer, mon but est pédagogique : il est d'essayer de dire si vous ne prenez pas en compte aujourd'hui la situation des étrangers, vous mettez en cause de manière générale les droits de l'Homme. »

C'est Le Monde du 9 mai qui évoque le thème de "la préférence nationale ": "Au coeur de la pensée d'extrême droite, la préférence nationale s'est doucement instillée au pays des Lumières... Chaque page du rapport (...) affirme cette réalité. " Et ce rapport serait " d'autant plus 
important qu'il concerne aussi les Français d'origine ", puisque, à ses yeux, "le respect des droits des étrangers est un marqueur essentiel du degré de défense et de protection des droits et libertés dans un pays".

Le quotidien rappelle la demande du Défenseur des droits adressée au ministre de l'Intérieur d'intervenir pour " empêcher la propagation d'interprétations du droit divergentes ou illégales au sein des préfectures " et de "procéder à des rappels réguliers du droit applicable ". " Sans certitude que la volonté politique soit là " ajoute Le Monde.

Pour Le Figaro (9 mai), "les étrangers sont victimes d'une "logique de suspicion" ». Blandine Le Cain écrit que "le rapport relève ainsi l'existence de deux phénomènes. D'abord, la marge de manœuvre importante laissée aux autorités afin de faire face aux différentes situations entraîne, dans ce contexte de méfiance, des décisions plus sévères que celles prévues par le droit. D’autre part, cette méfiance entraîne des cas de discriminations qui n'ont pas de fondement juridique mais perdurent en raison de l'état d'esprit actuel. "

Le 10 mai, Marianne titre "La France discrimine ses étrangers ". Sur la recommandation faite par le Défenseur des droits " au ministre de l'Intérieur de procéder à des rappels réguliers du droit applicable ", Clémence Barral interroge : " Mais cette disparité entre ce que dit la loi et son application est-elle une méconnaissance de ce que dit la loi ou des "oublis" volontaires? Cette question, le rapport Toubon ne se risque pas à y répondre. »

\section{Un enjeu démocratique}

Les "idées préconçues» et les «mythes» entretenus par un climat de suspicion et de peur nourrissent quelques libertés, un laisser-aller, voir un déni quant au respect des règles de droit en vigueur. Pour autant, le plus grave porte sur les atteintes aux principes juridiques mêmes qui fondent le pacte républicain, à commencer par le principe d'égalité. Le Défenseur des droits propose un travail d'édification et de déconstruction. II passe en revue les incohérences et les impasses des politiques (contrôle migratoire, tri entre "bons " et " mauvais » immigrés...), dénonce les idées reçues (comme le prétendu « appel d'air »), il interroge même ce que cachent les mots utilisés (migrants, réfugiés etc.). Et ici, le cidevant ministre de la Justice et directeur du Musée national de l'histoire de l'immigration est à son affaire quand il rappelle que "l'immigration est un fait social consubstantiel à la construction de la France et d'une partie de l'Europe " de sorte que la finalité des règles de droits ne devrait pas viser "la maitrise de l'immigration" ou "l'instauration d'un régime distinct" mais l'édiction et le respect "des droits" des étrangers. Pour Jacques Toubon, " cette logique de suspicion irrigue l'ensemble du droit français applicable aux étrangers arrivés récemment comme présents durablement - et va jusqu'à "contaminer" des droits aussi fondamentaux que ceux de la protection de l'enfance ou de la santé. (...) le fait que le droit et les pratiques perçoivent les individus comme "étrangers" avant de les considérer pour ce qu'ils sont, enfants, malades, travailleurs ou usagers du service public, conduit à affaiblir sensiblement leur accès aux droits fondamentaux ».

Pierre Rosanvallon aime à rappeler cette formule empruntée à Michelet : "Pour faire la démocratie, il faut faire cesser la terrible ignorance dans laquelle nous sommes les uns des autres. "C'est aussi ce que rappelle ce rapport sur les droits des étrangers : l'ignorance et les préjugés sur les étrangers interdisent de faire société en commun. 